\title{
Social Impact on Hybrid and Retrofitted Three-Wheelers
}

\author{
Sahil. S. Uttekar \\ Dept. of Mechanical Engineering \\ Army Institute of Technology \\ Pune, India \\ sahiluttekar4441@gmail.com
}

\author{
Sarvesh. S. Phatak \\ Dept. Electrical Engineering \\ Bharati Vidyapeeth(Deemed to be \\ University) \\ Pune, India \\ sarveshphatak@gmail.com
}

\author{
P. B. Karandikar \\ Department of E\&TC Engineering, \\ Army Institute of Technology \\ Pune, India \\ pbkarandikar@gmail.com
}

\begin{abstract}
To boost electric vehicles on Indian roads government brought up a policy that can eliminate personal vehicles in the upcoming decades. With an increase in shared mobility such as metros, monorails, and BRT systems, last-mile connectivity has already acquired the importance of never before. Bicycles and electric three-wheelers can fit this sector. Electrically operated three-wheelers will also gain importance in last-mile goods delivery. Similarly, the Internal Combustion Engine (ICE) based three-wheelers will pass through a phase of retrofitting and use of hybrid energy sources to fulfill economic and technological needs. It is very important to study retrofitting and hybrid three-wheelers in detail to understand how they can fill the gap of transition from ICE based system to a fully electrically operated model. These vehicles will have a social impact on consumers as they are cost-effective vehicles. Innovative, swapping of battery and fuel tank is proposed to address some of the needs in this transition. This paper presents techno-commercial aspects of retrofitted threewheelers and how to bring an economically viable hybrid version of three-wheelers.
\end{abstract}

\section{Keywords—retrofit, hybrid, three-wheeler, battery swapping}

\section{INTRODUCTION}

Online work has limitations and hence the movement of humans will be always important in any era. So far, there are three transportation modes i.e. Air, rail and road. For longer journeys and cargo, air transportation is the best. Amongst all, road transportation is most commonly used by all and in all kinds of climatic as well as geographical conditions. The road transportation of passengers or goods also offers a choice of two, three or four-wheelers. Three-wheelers faces stability issues at higher speed though they are the most popular mode of city transportation of goods and passengers. Passenger transportation is catered by auto-rickshaws and good's transportation is mainly handled by three-wheeler tempos in the majority of the small, medium, and big towns of developing and developed countries in the world. The need and design of three-wheeler tempos are different from auto-rickshaws. For long distance travel by road, fourwheelers are suitable as an option to rail or air. For last-mile connectivity it is three-wheelers which are more suitable. Three-wheelers are still an affordable means of short and medium distance for public transportation and last-mile cargo transportation. Taxi aggregators start-ups like (Ola, Uber, etc) are well established the importance and added cars to their products. Some start-ups operate as if aggregators in the good's transportation sector and finding it that they are opting for electric modes of transportation. In FY2019-20, more than six lacs three-wheelers i.e., goods carriers and auto-rickshaws were sold out in India and more than five lacs were exported. These figs can give idea to the reader about how many three-wheelers might be playing in all developing and underdeveloped countries. All such vehicles especially after vehicle age of 8 to 10 years can be retrofitted to make it operational in city areas where pollution is a big issue. The concept of hybrid vehicles needs to be incorporated into the tricycle market segment to meet the pollution requirements of the city and its exterior. It is possible to use renewable energy more effectively by hybrid three-wheeler vehicles [1].

Solar and wind energies are available in a specific time slot and to use them effectively hybrid vehicles can be effective [2],[3]. Hybrid vehicles have come in mid and large segments vehicles effectively [4],[5]. Many industries and researchers have come up with retrofitted and hybrid versions of vehicles using a fuel cell, compressed natural gas as well as electricity [6]-[9]. Retrofitting in three-wheelers is implemented without considering technical aspects and it is done by local companies [10],[11]. Many researchers have presented design, analysis, and simulation of retrofitted vehicles and have come up with many major aspects in it[12]-[15]. The use of a supercapacitor is the latest addition in it [16]. Many researchers have tried micro-hybrid and mild hybrid designs of electric three-wheeler considering only energy aspects [17][18]. After studying various kinds of literature on retrofitted vehicles and hybrid vehicles one major research gap was identified. Hybrid version and retrofit version of three-wheeler is not much seen in market. It is felt that there will be an entry of these types of versions in the three-wheeler segment handling passenger and goods movements. The detailed study of retrofit and the use of hybrid energy sources in three-wheelers are carried out and some interesting outcomes have been presented in this paper which will make it viable. There is an urgent need for some major companies to come into the market of retrofitting in the three-wheeler segment. As there will obvious impact on emissions, abandon on fossil fuels, and reduction in noise. The society can be turned into an eco-friendly environment nature. There is also a need of coming up with a hybrid three-wheeler. Details analysis of this is found as research gap which is addressed in this paper.

This paper is organized as follows, section 2 deals with the study of retrofitting in three-wheeler segments. Section 3 consists of the concept of a hybrid three-wheeler. Section 4 describes economic aspects and section 5 has concluding remarks.

\section{RETROFITTED THREE-WHEELER}

The number of four-wheelers in India is around 28.2 million approximately and the number is also similar in many other countries. This is mainly because of an increase in human wants and the usage of private cars as daily usage 
which has led to increase the pollution level. For the solution to these problem many governments are adopting electric vehicles. In India, some start-ups are coming with retro fitment kits for Internal combustion engine (ICE) vehicles. The usage of these kits can be done for deregistered vehicles or currently used vehicles. This will be more economical for consumer than buying a new one. A start-up E-Trio in India is one success story in this field. Most of the time lithiumion battery of (Nickel-Manganese-Cobalt) NMC or (lithiumion manganese-oxide) LMO type is used [19] with motor power of $10 \mathrm{kw} / 15 \mathrm{kw}$ in such retrofitted 4 wheelers as shown in Fig 1.

In India "Tempo" or "minibus" a four-wheeler type of vehicle is used for public transportation. The Mini Buses, as well as Tempos, are used in transportation for school students, software professionals, and picnics in most of the cases. The daily use of these vehicles is around $50-60 \mathrm{~km}$. If these vehicles are retrofitted it will be economical in refuelling as well as the overall maintenance cost of the vehicle will reduce. If swapping stations are implemented in those areas the swapping will take less than 5 minutes which will increase the running of the vehicle and charging of battery issue will also be sorted. However, the specification of the battery and motor can differ as per the customer's requirement. Therefore, the design of vehicles and seating capacity will be the same as the old one.

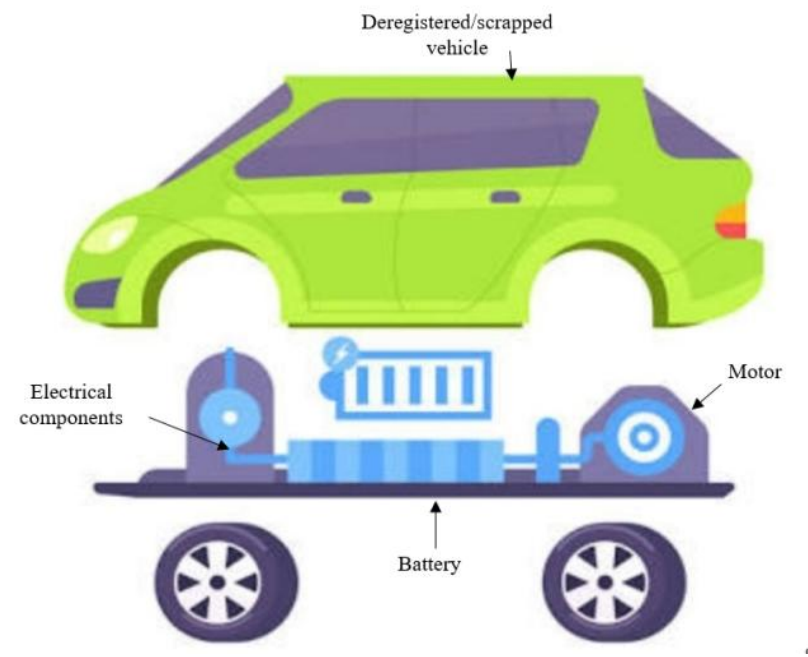

Fig. 1. Figure of Retrofitted 4-wheeler

The most found type of vehicle on the road is twowheeler. But, the number of two-wheelers on an Indian road is very close to figure of four-wheelers which is approx. 21.8 million. Most of the middle class people around the globe uses two-wheeler as their primary vehicle. Many small startups in a country like India are trying successful implementation of retrofitting of two-wheelers. In gear type of vehicles, the retrofitting can be done in such a way that the hub motor with a suitable diameter can be fitted in rearwheel and battery can go in place of the IC engine area. The disadvantage of this retrofitting is that the appearance of the vehicle is not the similar as the previous one. In retrofitting of non-gear two-wheeler the battery is fitted in boot space and the hub motor in the rear wheel. In this retrofitting the appearance of the vehicle is the same as the old one. A good number of start-ups in the retrofitting of two-wheeler will be seen in the coming decades.
The use of public transportation is the best way of communication of all time instead of using private vehicles. Most often used public transport vehicles in day to day life are Auto rickshaws. More than 2 million auto-rickshaws are there on Indian roads as of now[20]. Auto-rickshaws were manufactured as per BS-IV norms and as per the government "scrappage policy," the BS-IV vehicles will be dumped off. So, instead of dumping BS-IV vehicles (four-wheeler, twowheeler, and three-wheeler), they can be retrofitted to an electric one.

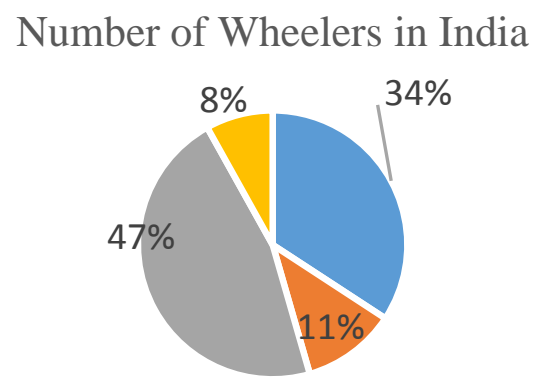

\section{- 2-wheeler 3-wheeler}

Fig. 2. Number of Wheelers in India

Data in Fig.2 represents the number of vehicles in various segments on Indian roads till 2018. Where twowheelers are 21.18 million, three-wheelers are 7.01 million, four-wheelers with 28.2 million[21] and Tempos/buses take 5 million on Indian roads. Thus, this figures will also increase day by day.

The retrofitting option can be well accepted by the enduser/ industry. The performance of the e-rickshaws can be matched with ICE rickshaws by studying different parameters. The solution can be cost-effective if it is available at about $50-60 \%$ price less than buying new autorickshaw. As there are no changes in body or suspension system the comfort level to driver and passenger is improved. Due to removal of ICE parts there is no vibration and sound like IC engine.

While retrofitting an Auto-rickshaw to E-rickshaw some major parts are in use and some are temporarily thrown off. Table 1 shows comparison of major parts in Auto-rickshaw and E-rickshaw.

TABLE I. PARTS COMPARISION OF AUTO-RICKSHAW AND ERICKSHAW

\begin{tabular}{|l|c|c|}
\hline \multicolumn{1}{|c|}{ Parts } & $\begin{array}{c}\text { Auto- } \\
\text { rickshaw }\end{array}$ & $\begin{array}{c}\text { E- } \\
\text { Rickshaw }\end{array}$ \\
\hline Frame and windscreen & $\checkmark$ & $\checkmark$ \\
\hline Seats & $\checkmark$ & $\checkmark$ \\
\hline Sunroof & $\checkmark$ & $\checkmark$ \\
\hline Motor & & $\checkmark$ \\
\hline Body & $\checkmark$ & $\checkmark$ \\
\hline LEDs/ Bulbs & $\checkmark$ & $\checkmark$ \\
\hline Chassis & $\checkmark$ & $\checkmark$ \\
\hline Battery and battery box & & $\checkmark$ \\
\hline Brakes & $\checkmark$ & $\checkmark$ \\
\hline
\end{tabular}




\begin{tabular}{|l|c|c|}
\hline \multicolumn{1}{|c|}{ Parts } & $\begin{array}{c}\text { Auto- } \\
\text { rickshaw }\end{array}$ & $\begin{array}{c}\text { E- } \\
\text { Rickshaw }\end{array}$ \\
\hline Suspension & $\checkmark$ & $\checkmark$ \\
\hline Controller/digital speedometer & & $\checkmark$ \\
\hline Tyre & $\checkmark$ & $\checkmark$ \\
\hline Fork & $\checkmark$ & \\
\hline Engine & $\checkmark$ & \\
\hline Gas cylinder & $\checkmark$ & \\
\hline
\end{tabular}

In retrofitting the most important parameter is balancing the weight of retrofitted auto rickshaw. Even though removing parts of the ICE which are not in use will not reduce a huge amount of weight on the vehicle, due to battery weight the weight of auto-rickshaw will be increase. But, using lightweight materials can reduce the weight as it will be economical in buying. Some parts are listed below which can be turned into lightweight components they are:-

The following methods can be used to reduce weight.

- Glass to be replaced with transparent plastic material named polycarbonate.

- Headlight/ tail lamp should be of LED type.

- Sunroof material to be Rexine.

- Mudguards to be of light-weighted plastic

- Wheels rim to be lightweight alloy material.

- Mirrors to be replaced by a camera screen.

- Seats to be of sponge or polyurethane.

- Body parts to be of fibre material.

- Use Digital speedometer

The most important problem faced by India while adapting E-mobility is the charging infrastructure of EV. There are many undeveloped areas in India which face scarcity of electricity. The charging infrastructure in India can be carried by use of renewable energy. The most found of renewable energy in India is solar and wind which can be used for charging EV batteries. Also at the battery "swapping station" the uncharged batteries can be recharged by renewable energy. So, customers can swap the batteries in less than 2 minutes by swapping it in eco-friendly manner. The charging of batteries can be done at home as the charger will be AC to DC supply. The customer can swap their batteries at home keeping an additional one. Also regenerative braking can be an additional feature to the kit, charges may differ. In future turbochargers will be in use and also off grid and on grid charging station will be implemented.

\section{HYBRID THREE-WHEEELER}

Nowadays there is a proliferation in the sector of electric vehicles across the globe. Electric vehicles are broadly classified into four types, a) Battery Electric Vehicle (BEV)It uses chemical energy stored in a rechargeable battery pack as the only source of energy. b) Fuel Cell Electric Vehicle (FCEV) - it uses a fuel cell, instead of a battery and maybe a supercapacitor for energy requirements. c) Hybrid Electric Vehicles (HEV) - it uses the minimum two types of energy sources like IC engine and electric source. d) Plug-in Hybrid Electric Vehicle (PHEV)- In this type of vehicle battery can be recharged in two ways i) Regenerative braking ii) Plug-in some external power source. Hybrid Electric vehicles concept is been developed in all types of vehicles i.e. two wheeler, three-wheeler, and four-wheeler. The hybrid fourwheeler concept is popular amongst all as high cost is not an issue for customers. The simple block diagram for the same is shown in Fig 3. Many international players are introducing hybrid four-wheelers in the market like Tesla, Volvo, Audi, BMW, Toyota, Honda, Mahindra and Mahindra, and Tata motors. Many hybrid two-wheeler players are present in the international market. The simple block diagram for the same is shown in Fig 4. Hybrid four-wheelers mainly in-corporate ICE and electric motor working in harmony for its propulsion. Normally the ICE capacity of an Auto-Rickshaw is different for different manufacturers.eg Bajaj RE is a $198 \mathrm{cc}$ vehicle wherein Piaggio Auto Rickshaw is 230cc vehicle. Both have power of about $8 \mathrm{~kW}$ currently, the Electric three wheeled variants in the market are also having different capacities of motor and battery. E.g. Kinetic Green Electric three-wheeler uses $48 \mathrm{~V}$ battery and has $1.9 \mathrm{~kW}$ BLDC motor for its propulsion while Mahindra Treo has a battery rating $48 \mathrm{~V}$ lithium-ion with an installed capacity of $7.37 \mathrm{kWh}$ to drive a $5.4 \mathrm{~kW}$ motor. The concept of plug-in hybrid three-wheeler can be operational with many effects on initial cost. By using proposed battery swapping stations and battery- Fuel tank swapping stations as shown in Fig 5, it can be made feasible. These hybrid three-wheeler will operate within a city with battery swapping stations. The conventional gas stations can add up to their infrastructure or could be replaced as a whole with a battery swapping station. In this the spare charged batteries are to be kept as a reserve and can be used at the time of swapping. These batteries can also be swapped with other type of vehicles if the overall specifications of the battery are matching. Sun mobility has already installed a battery swapping station in Bengaluru for two and three wheeled vehicles. Once the vehicle goes out of the city, its battery will be removed and a portable fuel tank will be joined with a fixed amount of fuel in it (this could be optional). Thus the owner of a hybrid three-wheeler will not have to bear the cost of battery as well as that of the fuel tank.

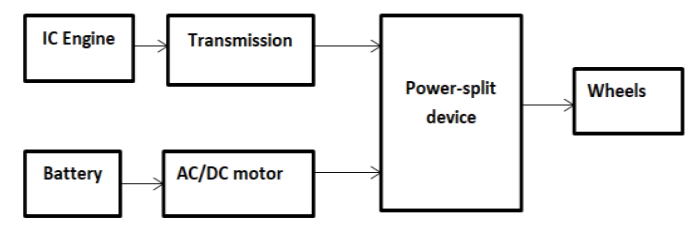

Fig. 3. Hybrid four-wheeler block diagram

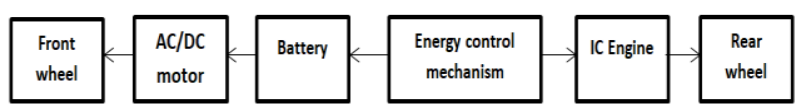

Fig. 4. Hybrid two-wheeler block diagram 


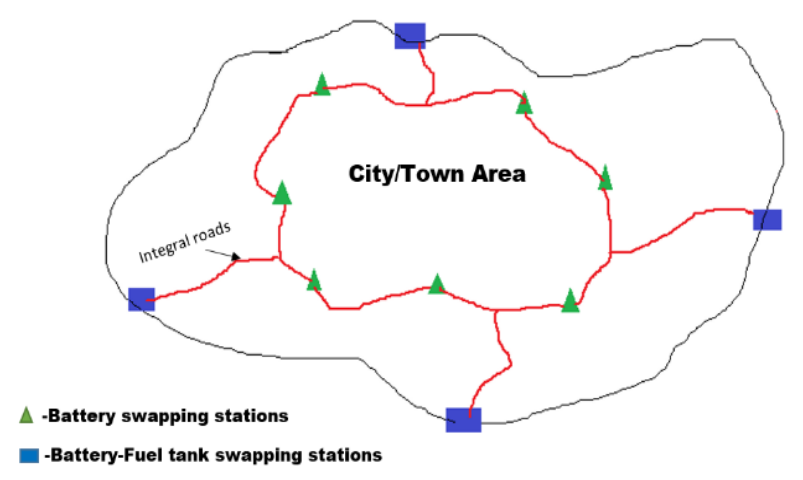

Fig. 5. Proposed Plug in hybrid three-wheeler plan for any city or town

Hybrid vehicles are classified as follows:-

a) Series hybrid: In this configuration the vehicle generally uses motor for driving the wheels. The engine is not directly coupled to wheels as shown in Fig 6. It is a simple drive train. Hence, it can be made to work purely electric by shutting the ICE when the battery is fully charged.

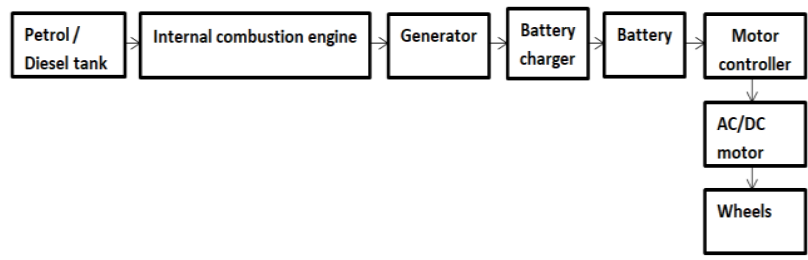

Fig. 6. Series Hybrid block diagram

b) Parallel hybrid: Here, both the sources can drive the wheels. Motor and engine are linked through transmission as shown in Fig 7. Motor can be used for low speed and ICE for high speed.

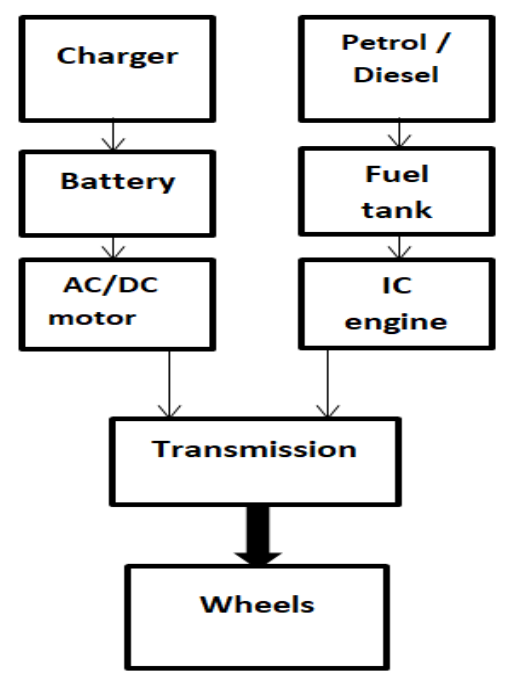

Fig. 7. Parallel Hybrid block diagram c) Series-parallel: It has features of both series and parallel. It is also called a combined hybrid or power split. In this configuration, there is a double connection between the engine and drive axle i.e. mechanical and electrical as shown in Fig 8.

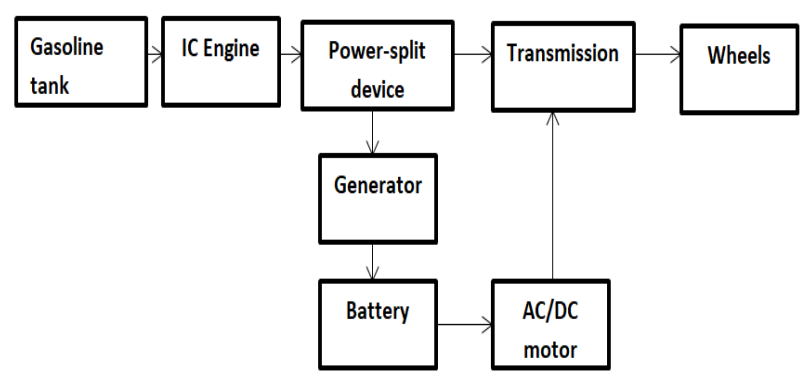

Fig. 8. Series-parallel Hybrid block diagram

Parallel and combined hybrid can be further classified according to the dominant portion (i.e. ICE or electric motor) in the following types

\section{A. Full hybrid}

A full hybrid runs only on engine, just the motor or combination of both. A large capacity battery pack is needed for battery-only operation. ICE will shut when the battery is sufficient to provide the power.

\section{B. Medium hybrid}

They use ICE for primary power with a motor connected in parallel. EV mode is possible for a limited period of time. Battery size is small so power small.

\section{Micro hybrid}

It consists of ICE and an electric motor in a parallel hybrid configuration. It allows ICE to be turned off during braking, coasting, or stopping. Hence, employing regenerative braking but cannot operate on an electric-only mode of propulsion.

\section{Mild hybrid}

It cannot run fully on Electric motor but it can give extra power to ICE by a motor. The small size of the battery is used and hence, low cost.

Considering in three-wheeler segment, a three-wheeler needs to be economical for a driver and should cover a longrange in the congested city area. Hence, a medium hybrid configuration can be employed for a limited range. Hybrid three-wheeler will be very successful as it will reduce $\mathrm{CO}_{2}$ emissions, will have more range due to the parallel working of ICE and electric motor. For a tempo, one may go for a full (strong) hybrid type of configuration.

Hybrid sources of energy-Hybrid three-wheeler will use the battery as its main source of power and the battery module can be charged in two ways First, the on-board plugin battery charger. Second, PV panel and third, ultra capacitors. The battery is fully charged by plug-in battery charger through grid and PV panel or Ultra capacitor try to keep the state of fully charged battery module by continuous trickle charring as shown in Fig 9. 


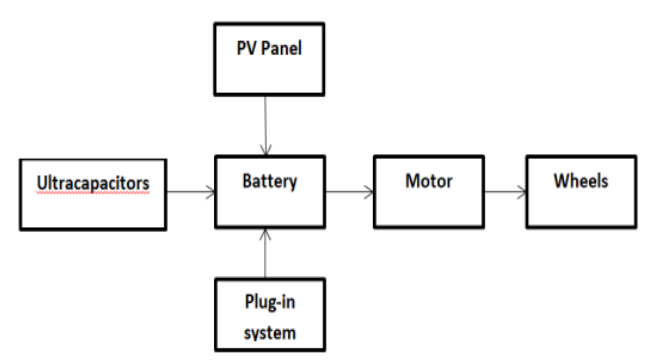

Fig. 9. Hybrid vehicle block diagram with various energy sources

\section{ECONOMIC ASPECTS}

The cost of an auto-rickshaw in India is approx. 1.6lac and a Tempo costs around 6.95lac. Both costs are too high for ordinary people when they buy it. But these costs will be zero at the time of deregistration. The retrofitting concept will not only enhance the life of the vehicle but also the new retrofit vehicle will be economical. The retro fitment cost for Auto rickshaw will be around 90k (excluding registration and government charges) this cost is $30-40 \%$ economical than Auto rickshaw.. The retrofitting option can be well accepted by the end-user/ industry/ subjected. The performance of the e-rickshaw is matching with Auto rickshaw. The solution is cost e-effective if available at about $50-60 \%$ price less than buying new E-rickshaw. Since there is no change in body or suspension system, etc. the comfort level to driver and passenger is improved as there is no vibration, sound, and driving like an IC engine. Battery with higher changes in 5000 cycles, will give a peace of mind and cost savings for a longer period, by little cost addiction. Even with a top-up charge during breaks, the battery will be more than 8 yrs. The retrofitting of auto-rickshaws will close some business houses and will create opportunities for new businesses. The Retrofitting concept can pave the way for many aspiring entrepreneurs who find the EV industry a promising business prospect At the national level need for copper will increase as all retrofitted auto-rickshaws will use an electric motor which will have 2 to $5 \mathrm{~kg}$ copper winding in it. Aluminium may be a solution to it as India is dependent on other countries for copper. Lithium business will flourish but eventually, it will be import oriented at the first stage. Recycling of lithium will be a new type of business open for start-ups in the next decade. As many retrofitted vehicles will use a lead-acid battery, its business will see a lot of growth for the next 20 years. It will also provide encouraging Job opportunities for the skilled personnel. To release this load on the automotive industry, the retrofit plays an important role. Also in case of operational cost of battery swapping stations (BSS), the BSS owner could minimize its operation cost for battery charging/discharging by operating at a leastcost schedule, and further make a profit by participating in electricity market and offering extra services. In terms of the cost of real estate, as the BSS owner does not need access to spacious parking lots, substantial cost savings would be guaranteed. Power system operators will also benefit from this scheme as the BSS can be potentially used as a large and flexible resource for network congestion and peak load reduction [22]. Therefore, it directly suggests that there is vast scope for retrofit in the Indian industry as every year there will be rickshaws that will be deregistered. The industry would flourish and the economy would flow in rapidly due to this innovation.

\section{CONCLUSION}

The cost of a hybrid three-wheeler is higher than the conventional ICE three-wheeler due to the inclusion of battery, motor and control systems. In cities wherein one requires low speed, a hybrid three-wheeler can operate in electric mode and on highways where pollution is not an issue; it can switch to the mode of ICE. Addressing the extra cost is very important to make it feasible for a three-wheeler owner. It is recommended that the battery swapping concept in city area can reduce the cost of battery on the driver of three-wheeler i.e. both auto-rickshaw and tempo. It is proposed that there can be fuel tank-battery swapping stations at the borders of town/ city. This model will be most suitable for industrial cities like Pune, Surat, manufacturing hub cities like Kanpur, Hyderabad and will also be beneficial for agricultural hub cities like Ludhiana etc. This concept will push hybrid three-wheelers which can help cities to address the issue of pollutions to some extent and make three-wheeler operating costs very low. Thus battery swapping stations in the city and battery-fuel tank swapping station outside the city is the proposed model of operation. This model will suit retrofitted vehicles also. Apart from this, the cost of a hybrid three-wheeler will remain within reach of the owner. Society will be using eco-friendly vehicles which will help our diversity in future.

\section{ACKNOWLEDGMENT}

Authors would like to thank Mr Nandu Sarolkar of M/S Sarvesh Engineers, Pune for useful discussions.

\section{REFERENCES}

[1] S. M. Lukic, P. Mulhall and A. Emadi, "Energy autonomous solar/battery auto rickshaw", J. Asian Elect. Vehicles, vol. 6, no. 2, pp. 1135-1143, Dec 2008.

[2] Malik Sameeullah, "Design of global MPPT controller for photovoltaic system and application to solar hybrid energy system", 2015 .

[3] M. Sameeullah and S. Chandel, "Design and analysis of solar electric rickshaw: A green transport model", International Conference on Energy Efficient Technologies for Sustainability (ICEETS), pp. 206211, 2016.

[4] O. D. Momoh and M. O. Omoigui, "An overview of hybrid electric vehicle technology", IEEE Vehicle Power and Propulsion Conference, pp. 1286-1292, 2009

[5] P. Mulhall, M. Naviwala, S. M. Lukic, J. Braband and A. Emadi, "Entrepreneurial projects program at Illinois Institute of Technology: Solar/battery hybrid three-wheel auto rickshaw for India", pp. 682689, September 2007.

[6] Brian Surgenor and Brant Peppley, "Effect of Control Strategy on the Performance of a Fuel Cell Hybrid Electric Auto Rickshaw", vol. 1, July 28, 2018.

[7] Yusuf Gurkaynak, Omer Onar, "A Photovoltaic-Battery Powered AllElectric Rickshaw for the Indian Market," SAE International by University of Minnesota, August 01, 2018.

[8] Mehta. R, Shah, P., Gupta. H, Bhat, "Conversion of a CNG Powered Auto Rickshaw to an Electric Rickshaw Designed for Indian Conditions," SAE Technical 2014

[9] Madhav G. Badami and Conor C O Reynolds, "Auto-rickshaws in Indian cities: Public perceptions and operational realities", vol. 4, pp. 1-3, July 2018.

[10] Kiran B Pattar, Praveen Kumar R H, Rahul Raj K Jain, Sharanbasappa S Patil, "Methodology for Retrofitting Electric Power Train in Conventional Powertrain-based Three-wheeler", vol. 5, pp. 263-270

[11] Madhu Sivaraman, "Electric Rickshaw,"CPPR Policy Brief Series II, vol. 1, pp. 1-7, 19 June 2014.

[12] Dhvani Pandya and A.M Bisen, "Analysis of Chassis in 3-Wheeler E-Rickshaw for weight reduction”, vol. 4, pp. 1-3, July 2018. 
[13] Venkata Mangaraju Karanman and Ashitawa Ghosal, "Studies on wobble mode stability of a three wheeled vehicle", vol. 227, pp. 1200-1209, August 2013.

[14] Chikesh Rajan, J. Akthar, Akshay Kumar Tiwari, Neetenjay Kumar Choubey, Roshan Kumar, Lalchand Rajwan, Nitesh Kumar and Rajbalabh Kumar, "Desgin and Fabrication of Tri Rickshaw with solar charging staion”, vol. 8, pp. 358-366, July 2017.

[15] Shashank Singh, "A study of the battery operated e-rickshaws in the state of Delhi", Researching reality summer Internship 2014, working paper 323, pp.1-32.

[16] Amari Mansour, Chabchoub Mohamed Hedi and Bacha Fazoui, "Experimental study of a pack of super capacitors used in electric vehicles", vol. 2017, pp. 1-8, 14 August 2017.

[17] Subhasachi Saha, Souvik Roy, Md. Sazid Rhaman and Md Zahid Hassan, "Micro-hybrid with Ultra-Capacitors Based Three-Wheeler", vol. 1, pp. 69-79, April 2018.
[18] Sagar Tatipamula, "Study of parallel electric hybrid three wheeled motor taxi,'International conference on green computing and technology, vol. 3, pp. 38-41, May 2009.

[19] Yu Miao, Patrick Hynan, Annette von Jouanne, Alexandre Yokochi, "Current Li-Ion Battery Technologies in Electric Vehicles and Opportunities for Advancements", vol. 1, pp. 2-20, March 2019.

[20] https://www.wri.org/news/2012/02/media-alert-launch-new-reportauto-rickshaws-india

[21] http://www.knowindia.net/auto.html\#: :text=Domestic\%20motor\%20 vehicle $\% 20$ sales $\% 20 \mathrm{in}$, and $\% 2021.18 \% 20 \mathrm{~m} \% 20$ two\%2Dwheelers

[22] Mohsen Mahoor, Zohreh S. Hosseini, Amin Khodaei, "Least-cost operation of a battery swapping station with random customer requests", vol.172, pp. 913-921, 1 April 2019. 